\title{
Additional Space Dimension in Bound Spaces Created by a Central Object
}

\author{
T. V. B. S. Satyanarayana Murthy \\ Civil Design Department of Power Projects Division, BGR Energy Systems Limited, Chennai, India \\ Email: tvbssmurthy@gmail.com
}

Received 16 November 2014

\begin{abstract}
Space is a common entity in any static or dynamic system of objects whether the system is a bound system or an open system. Space is described by either measured or abstract dimensions. All motions, momentum and energy transfers take place in space in any given system of objects. By understanding space we understand many physical phenomena happening in space. One method is to observe similarities among phenomena, deduce possible relations and validate the relations through known results. De Broglie wavelength for matter waves is a typical theorized abstract dimension existence of which is established through experiments later. In this paper, the author studies two bound spaces in two bound systems, namely, atomic bound systems and gravitationally bound systems. Both these bound systems have similar characteristics; they have inertial masses in motion with constant kinetic energies for a given orbital distance around the respective central object. In atomic bound space, the central object is the central positive charge which plays the role of creating the bound space around it. In gravitationally bound space, it is the central mass that plays the same role. Thus for these two bound systems a common constitutive relation between the energies of inertial masses in kinetic state, their distance from central object could be present. By noticing the similarities of the two systems, the author proposes such a relation through introduction of an additional space dimension. The existence of the proposed additional dimension is proved in this paper by considering hydrogen atom for atomic bound space and by considering any gravitational system for gravitationally bound space. Though the magnitude of the additional space dimension is different in both the situations, the additional space dimension exists. It is observed that in hydrogen atom the additional space dimension is a constant for the given positive charge of hydrogen atom when electron is in any energy state having principal quantum number of any value from 1 to 5 and with the orbital quantum number zero. For other quantum numbers additional space dimension exists. In the case of gravitational bound space, the additional space dimension is constant for a given mass of the central object for any energy of orbiting inertial mass. The author concludes that total mass energy of an inertial mass having a constant kinetic energy in any bound space is related to an additional space dimension defined by the constitutional property of the central object creating that bound space. As the relation is generic, it throws opportunity to examine other known similar macro, micro or quantum bound spaces created by central objects with different constitutive properties.
\end{abstract}

\section{Keywords}

Central Object, Bound Space, Gravitational, Charge, Additional Space Dimension, Energy, Mass 


\section{Introduction}

Einstein's mass energy equivalence derived from special relativity [1] is universal conservation law. The equivalence basically relates the total energy of an inertial mass in motion. In some bound spaces, typically the inertial masses are in kinetic state undergoing either oscillatory vibratory motion or orbital motion about a central object. The central object is a central positive electric charge in a quantum bound space like atom and the central object is a gravitating mass in gravitationally bound space. The constituent properties of the central object control the space around it. In case of gravitational bound space, Einstein's general relativity [2] gives the effects of central mass on space time curvature around it. In a gravitational system the kinetic energy of an orbiting inertial mass is governed by gravitational interactions. The energy of orbiting mass can have only definite value and satisfies Newton's law of universal gravitational attraction between masses [2]. In quantum systems like atomic systems, the electrons are in a kinetic state around the central nucleus containing positive charges. The energies of electrons are quantized. The energies satisfy Coulomb's law of attraction between opposite electric charges [2]. Typical characteristic of a stable bound state is that the kinetic energy of an inertial mass attached to the system at a given distance from the central object is constant with time, and thus the kinetic energy of the inertial mass is quantized. In both the bound spaces the energies are quantized. However, in spite of vast knowledge accumulated on gravitational and quantum stable bound systems, no formal common physical relationship is available. In this paper, the author intuitively identifies a relationship between energy and an additional space dimension in these bound spaces where a central object creates the bound space. The results indicate that wherever quantization of energy prevails, an additional space dimension is present which is directly related to constitutional properties of the central object and that the dimension is independent of the properties of the inertial mass in kinetic state under the effect of central object. The results show that the additional space dimension is constant for a given stable bound state. The theory opens up a new reliable basis to understand physical phenomena related to different inertial mass systems with different central objects having different constitutive properties.

\section{Additional Space Dimension}

Let us consider a stable bound system with a central object. Let an inertial mass having rest mass $m_{0}$ be present in oscillatory or orbital motion around the central object at a distance, $D$. Let the kinetic energy of the inertial mass be $K$ and total energy of the inertial mass be $E$. Then

$$
K=E-E_{0}
$$

where $E_{0}$ is the rest mass energy given by,

$$
E_{0}=m_{0} c^{2}
$$

where $c$ is the speed of light in vacuum.

For the above bound system, the author proposes

$$
E=E_{0}\left(1+\delta_{b} / 2 D\right)
$$

where $\delta_{b}$ is the additional space dimension for the found system.

We further assume that $\delta_{b}$ is dependent on the constitutional properties of the central object which controls the bound space and holds the bound system intact. We find out the value of $\delta_{b}$ for hydrogen atom and gravitational system. By simplifying Equation (3) we get,

$$
\delta_{b}=2 K D / m_{0} c^{2}
$$

\subsection{Electron in Hydrogen Atom}

First, we consider the quantum bound system i.e. the atomic system, where the central object is electrically charged nucleus. Let us consider an electron with rest mass, $m_{e}$ in kinetic state around the central charge. At quantum level it is highly difficult to have precise value of $D$ required in Equation (4). For hydrogen atom the distance $D$ is available from Schrodinger's wave equation [1] in the form of expected radius, $\overline{r_{n l}}$. Hence we consider hydrogen atom to validate the relationship. 


$$
\begin{gathered}
E_{0}=m_{e} c^{2} \\
E=K+m_{e} c^{2} \\
D=\overline{r_{n l}}
\end{gathered}
$$

The kinetic energy of electron depends on the quantum number $n$ as given by

$$
K=\mu Z^{2} \mathrm{e}^{4} /\left(4 \pi \varepsilon_{0}\right)^{2} 2 \hbar^{2} n^{2}
$$

In (8) $K$ is in $\mathrm{eV}$ and $n$ is the principal quantum number and $Z=1$ for hydrogen atom. The expected values of the distance of electron from nucleus $\overline{r_{n l}}$ are given by the equation given in [1],

$$
\overline{r_{n l}}=\left(n^{2} a_{0} / Z\right)\left[1+(1 / 2)\left\{1-l(l+1) / n^{2}\right\}\right]
$$

where $a_{0}=4 \pi \varepsilon_{0} \hbar^{2} / \mu \mathrm{e}^{2}$ is radius of smallest orbit of Bohr's hydrogen atom and $l$ is orbital quantum number. By substituting for $K$ from Equation (8) and $\overline{r_{n l}}$ from Equation (9) in Equation (4), after simplifying we get

$$
\delta_{b}=A \mathrm{e}^{2} / 4 \pi \varepsilon_{0} m_{e} c^{2}
$$

where

$$
A=1+(1 / 2)\left\{1-l(l+1) / n^{2}\right\}
$$

The values of $A$ are as follows.

$A=1.5$ for $n=1,2,3,4,5, \quad l=0$ where the energy of electron in these bound states is a constant. In other quantum states of electron, we have $A=1.25$ for $n=2, l=1 ; A=1.39$ for $n=3, l=1$; and $A=1.17$ for $n=3, l=2$.

We find that $A=1.5$ is constant for energies of electron for any $n$ and $l=0$ states and hence the space dimension $\delta_{b}$ is a constant in those bound states in hydrogen atom. In other states the additional dimensions exist and in hydrogen atom the additional dimension is influenced by the central charge.

\subsection{Gravitationally Bound Space}

Second, we consider any gravitationally bound space. In gravitational system, as we know the central object is a gravitating mass. We define the central object has a gravitating mass $M_{0}$ equal to its inertial mass. We consider any orbiting object having an inertial mass $m_{0}$. Let the distance of orbit of orbiting object be $r$. Thus for the orbiting object we have

$$
\begin{gathered}
E_{0}=m_{0} c^{2} \\
E=K+m_{0} c^{2} \\
D=r
\end{gathered}
$$

The kinetic energy of any the planet from Newton's gravitational force law is given by

$$
K=G M_{0} m_{0} / 2 r
$$

where $G$ is the universal gravitational constant.

Substituting for $K$ from Equation (15) in Equation (4) we get the additional dimension for bound space controlled by mass of the central object as

$$
\delta_{b}=G M_{0} / c^{2}
$$

Thus for a gravitational system the additional space dimension is a constant and is influenced by the mass of central object creating the bound space.

\section{Conclusion}

The additional dimension $\delta_{b}$ in Equation (4) is a generic dimension pertaining to a given bound space created 
by a central object. The additional dimension is related to the constitutive properties of the central object.

\section{Acknowledgements}

The author gratefully acknowledges the permission granted by the management of BGR Energy Systems Limited, Chennai, India, to publish this work.

\section{References}

[1] Eisberg, R. and Resnick, R. (2010) Quantum Physics of Atoms, Molecules, Solids, Nuclei, and Particles. 2nd Edition, John Wiley \& Sons Ltd., Wiley India (P) Ltd., New Delhi.

[2] Walker, J. (2011) Halliday/Resnick Fundamentals of Physics. 8th Edition, John Wiley \& Sons Ltd., Wiley India (P) Ltd., New Delhi. 